\title{
An improved workflow for accurate and robust healthcare environmental surveillance using metagenomics
}

\section{Jiaxian Shen}

Northwestern University https://orcid.org/0000-0003-4929-8955

\section{Alexander McFarland}

Northwestern University https://orcid.org/0000-0002-1803-3623

Ryan Blaustein

National Institutes of Health

\section{Laura Rose}

Centers for Disease Control and Prevention

\section{K. Perry-Dow}

Centers for Disease Control and Prevention

\section{Mary Hayden}

Rush Medical College https://orcid.org/0000-0002-4603-8501

\section{Vincent Young}

University of Michigan Medical School https://orcid.org/0000-0003-3687-2364

Erica Hartmann ( $\sim$ erica.hartmann@northwestern.edu )

Northwestern University https://orcid.org/0000-0002-0966-2014

\section{Article}

\section{Keywords:}

Posted Date: February 15th, 2022

DOI: https://doi.org/10.21203/rs.3.rs-1303703/v1

License: (1) (1) This work is licensed under a Creative Commons Attribution 4.0 International License. Read Full License 
An improved workflow for accurate and robust healthcare environmental surveillance using metagenomics

Jiaxian Shen ${ }^{1}$, Alexander G. McFarland ${ }^{1}$, Ryan A. Blaustein ${ }^{2}$, Laura J. Rose ${ }^{3}$, K. Allison PerryDow $^{3}$, Mary K. Hayden ${ }^{4}$, Vincent B. Young ${ }^{5}$, Erica M. Hartmann ${ }^{1, *}$

${ }^{1}$ Department of Civil and Environmental Engineering, Northwestern University, USA

$8 \quad{ }^{2}$ National Human Genome Research Institute, National Institutes of Health, USA

$9 \quad{ }^{3}$ Centers for Disease Control and Prevention, USA

$10{ }^{4}$ Department of Internal Medicine, Division of Infectious Diseases, Rush Medical College, USA

11 5epartment of Internal Medicine/Division of Infectious Diseases, The University of Michigan

12 Medical School, USA

13 *Correspondence: Erica M. Hartmann; 2145 Sheridan Road, Tech A322, Evanston, IL 60208-

14 3109; Email: erica.hartmann@northwestern.edu; Tel: 847-467-4528

\section{Abstract}

16 Effective surveillance of microbial communities in the healthcare environment is increasingly

17 important in infection prevention. However, current workflows are insufficient for proper risk

18 assessment. Upon evaluating and optimizing techniques, we recommend best practices and

19 introduce a well-structured workflow for metagenomics-based environmental surveillance that is

20 appropriate for low-biomass samples, distinguishes viability, and is quantitative. The workflow

21 was developed using a representative microbiome sample, which was created by aggregating 120

22 surface swabs collected from a medical intensive care unit. We recommend liquid-liquid

23 extraction, propidium monoazide treatment coupled with internal standards and absolute

24 abundance profiling (e.g., using qPCR), and a machine learning-based model for sequencing

25 depth calculation. In addition, whole-cell filtration and cultivation may be valuable under

26 particular circumstances. This workflow will contribute to more accurate and robust

27 environmental surveillance and infection prevention. Lessons gained from this study will also

28 benefit the continuing development of methods in relevant fields. 


\section{Introduction}

30 Effective microbial surveillance in the built environment is increasingly important in infection prevention, given the persistence of pathogens in environmental reservoirs and their potential transmission to patients ${ }^{1-7}$ (e.g., carbapenem-resistant Klebsiella pneumoniae in sink drains ${ }^{8,9}$ ). Furthermore, with the emergent studies showing synergistic relationships among pathogens on hospital surfaces ${ }^{10}$ and the possibility for pathogenic bacteria to acquire antibiotic resistance genes from non-pathogenic neighbors ${ }^{11}$, it is necessary to expand from targeted surveillance to untargeted methods. Untargeted methods are advantageous in identifying novel or rapidly emerging pathogens ${ }^{12}$. Metagenomics-based techniques are the most promising option to achieve these goals but are currently challenged by several limitations: 1) they are not powerful enough to extract valid signals out of the background noise for low biomass samples; 2) they do not distinguish between viable and non-viable organisms; and 3) they do not reveal the microbial load quantitatively ${ }^{13,14}$.

42 For challenge 1), adoption of appropriate negative controls has been emphasized ${ }^{15,16}$, along with 43 various bioinformatic filtering tools to remove putative contaminants ${ }^{17,18}$. While current efforts have largely focused on contamination prevention, increasing the biomass itself remains understudied $^{12}$. Having adequate biomass is essential, as previous work has indicated that a small amount of starting material (i.e., DNA) has adverse impacts on the outcome regardless of sample processing methods ${ }^{19}$. In practice, methods have been adopted as temporary fixes, such as pooling samples from different sites or dates ${ }^{20}$, and using wipes instead of swabs as sample collectors ${ }^{1}$. However, these workarounds are not always available ${ }^{21}$. Moreover, systematic evaluation and benchmarking of optimization strategies for metagenomic sample preparation remain largely unexplored.

52 For challenge 2), propidium monoazide (PMA) is the most widely used viability indicator compatible with molecular techniques. Though intensively optimized ${ }^{22}$, its efficacy and applicability in combination with metagenomics are controversial. A semi-quantitative systematic evaluation concluded that PMA treatment coupled with 16S rRNA gene amplicon sequencing (PMA-Seq) is reliable when the microbial community is not very complex, while uncertainties increase dramatically with complexity ${ }^{23}$. The uncertainties come from both heterogeneity of microorganisms (e.g., cell envelope structure differences, spore formation), and complexity of the background matrix (e.g., turbidity, salt concentration, dead cell density) ${ }^{13,24,25}$. While the microbial communities to be surveilled have their inherent advantage of being low complexity, little is known about the effectiveness of incorporating PMA with multi-species internal standards. To be appropriately rigorous, comparisons are needed relative to standard surveillance that does not consider viability (i.e., no PMA), as well as traditional methods (i.e., cultivation).

For challenge 3), pitfalls of using relative abundances in microbial profiling have been widely indicated. Such pitfalls include but are not limited to lack of unique connections between biological interpretations and experimental observations and unreliable comparisons across samples ${ }^{14,26,27}$.

67 Strikingly, mis-selection of analytical tools for relative abundance data could lead to as high as $68100 \%$ false discovery rates ${ }^{26}$. Besides flow cytometry ${ }^{28,29}$, combing sequencing with quantitative 69 PCR (qPCR) and including internal standards ${ }^{30}$ are two major means of making quantitative 
estimations out of next-generation sequences. In practice, previously reported applications for qPCR include air and dust samples in classrooms ${ }^{31}$; for internal standards, applications include Amazon River plume ${ }^{32}$, soil ${ }^{33}$, and stool samples ${ }^{34}$. However, comparisons are not yet available between metagenomics coupled with qPCR and with internal standards using low biomass environmental samples in the immediate vicinity of humans (e.g., healthcare settings).

An additional practical challenge in developing a robust pipeline with metagenomics is how deep one should sequence. While useful in whole genome sequencing, recommendations of coverages expressed by folds of genome sizes (e.g., $15 \mathrm{X}$ to $60 \mathrm{X}$ ) are not readily transferable to metagenomic sequencing (MetaSeq), as reads do not equally distribute across members with substantially different abundances. Nonpareil, a redundancy-based tool, estimates and projects abundanceweighted average coverage for metagenomics (expressed in percentage ${ }^{35-37}$. This helps reduce erroneous interpretations out of metagenomic results. Yet expected coverage is still largely unpredictable before sequencing is run. Researchers usually rely on previous experience of similar samples and the available budget to determine the sequencing effort (read size, unit: bp), which may lead to either a coverage too low, thus limiting the extractable information ${ }^{11}$, or a waste of resources ${ }^{1}$.

To address these challenges, we present a workflow for metagenomics-based environmental surveillance that is appropriate for low-biomass samples, distinguishes viability, is quantitative, and estimates sequence resources (Fig. 1). Liquid-liquid extraction, PMA treatment equipped with internal standards and absolute abundance profiling, qPCR, and a machine learning-based model are the recommended components for the comprehensive workflow, with whole-cell filtration and

91 cultivation as optional accessories.

\section{Results}

\section{Liquid-liquid extraction improves the power of handling low-biomass samples} To improve DNA yield of low biomass samples, we first compared 3 categories of extraction methods. Bead-beating and heat lysis followed by liquid-liquid extraction was the optimal method, as opposed to widely used column- and magnetic bead-based methods ("Methods", Supplementary Fig. 2) ${ }^{38,39}$. Notably, no detectable DNA was recovered using Qiagen DNeasy PowerSoil Kit. Supplementary to the recommendation that DNA input $\geq 1$ ng for Nextera Flex Library Prep kit, we correlated it to the practical outcome and found that DNA $>11.2 \mathrm{ng}$ corresponded to raw reads $>1$ e +05 (Supplementary Fig. 2).

101 In addition to being low biomass, environmental samples of interest are usually in the immediate 102 vicinity of humans, and thus often contain eukaryotic cells. These cells may compete with bacteria 103 for sequencing depth, lowering detectable resolution on bacteria, especially for low-abundance 104 members. Collection methods such as swabs and wipes can further recover abiotic debris along with biological materials, to which chemicals potentially interfering with downstream experiments may adsorb ${ }^{40}$. To address these issues, we evaluated the implementation of whole-cell filtration in

107 the workflow. Four filtration steps $(100,80,41,5 \mu \mathrm{m})$ were conducted in descending order of pore 
sizes. For our samples, filtration did not exert a significant effect on detected proportions of bacteria (Fig. 2a) or eukaryotic reads (Supplementary Fig. 3), according to paired tests ( $p \geq$ $1100.05)$. Considering that the non-bacterial proportion of our samples was relatively small $(\sim 1 \%)$, 111 filtration appears ineffective (or unnecessary) in increasing the bacterial proportion by excluding 112 eukaryotic cells for samples with similar characteristics. Instead, most of the eukaryotic reads were human-associated and thus able to be removed in silico (Supplementary Fig. 3). Moreover, we did not observe an increase in the number of rare taxa post filtration. Nevertheless, filtration did not negatively affect the number of recoverable taxa (Supplementary Fig. 4) ${ }^{41,42}$.

116 As expected, filtration introduced biomass loss of $\sim 13-44 \%$, according to 16S rRNA gene copy number (Fig. 2b). The biomass loss may be compensated by a two-fold concentration, material permitting. Alternatively, the total biomass loss can be reduced in practical applications where one-step filtration is streamlined. Filtration did not impact the overall bacteria composition (Fig. 2c), nor did it change the relative abundances of top abundant taxa (average abundance $>1 \%$ ) (Supplementary Fig. 5). This evidence supports the validity of using filtration to concentrate bacterial samples in sequencing-based experiments for profiling relative abundances. However, the absolute abundances would be affected disproportionately, as the extent of biomass loss varied across samples (Fig. 2b). Therefore, when an absolute metric is of interest, the recovery rate needs to be rigorously measured. Bacteria retention profiles on $5 \mu \mathrm{m}$ filters were similar to those of the liquid samples. However, bacterial members were not proportionally retained by filters of a larger pore size $(100,80,41 \mu \mathrm{m})($ Fig. $2 d)$. Hence treating microbial samples with large pore-size filters may introduce biases, even when relative abundances are used.

Taken together, for samples whose non-bacterial proportion is small (e.g., $\sim 1 \%$ ), it is unnecessary to incorporate filtration to increase the bacterial fraction. Filtration is valid in concentrating samples. However, for low biomass samples which are low in both cell density and quantity, 132 biomass loss outweighs the slight increase of bacterial signal. Instead, switching to a high-yield 133 DNA extraction method, such as liquid-liquid extraction, can achieve higher folds of signal 134 improvement (DNA concentration from undetectable to $18.62 \pm 1.16 \mathrm{ng} / \mu \mathrm{L}$ ).

\section{PMA and cultivation improve the ability to determine viability}

136 We examined the efficiency of PMA treatment coupled with metagenomic sequencing (PMA137 MetaSeq) on hospital-associated surface samples with the ZymoBIOMICS Microbial Community 138 spike-in as the internal standard. The Zymo community consists of 8 bacterial species and 2 yeasts, 139 which presumably will function more comprehensively and accurately regarding bias correction 140 and quality control than a single-species standard ${ }^{13,23,24,43}$. Sequencing outcomes were compared 141 with cultivation results for benchmarking, as cultivation is the gold standard for determining 142 microbial viability.

143 Absolute abundance of samples decreased after PMA treatment, indicating the depletion of non144 viable signals (Fig. 3c). This was further supported by the observation that $\boldsymbol{\alpha}$ diversity was lower 145 in PMA-treated samples (Fig. 3a), and that inter distances between paired samples were larger 146 than intra distances within each sample group (Jaccard Distance; Fig. 3b). We note that absolute 147 abundance should be used when analyzing sequence data involving viability assessment, as 
relative abundance profile is likely distorted (Fig. 3c-d $)^{33}$. Although relative abundance is informative in demonstrating presence/absence, it neglects the amount of overall biomass and thus may inflate the apparent abundance of even low-abundance organisms. While absolute abundance is more reflective of reality, field trials are necessary to determine whether absolute or relative abundance, or either, can be linked to infection or other clinical outcomes.

We calculated the efficacy of 8 spike-in bacteria ${ }^{23}$. The efficacy should be 1 under ideal conditions, given that the percentage of viable microbes in the Zymo community is negligible (Supplementary Fig. 6). The efficacy equaled 1 for all taxa, suggesting that PMA treatment is effective in low biomass samples regardless of taxonomy. This conclusion is partly consistent with our previous evaluation of PMA-Seq where E. coli was spiked in ${ }^{23}$.

158 Focusing on specific taxa (Fig. $\mathbf{4 b}$ ), we observed occasions of a complete depletion for highabsolute-abundance taxa and retention for low-absolute-abundance taxa, suggesting an effective viability distinction. Relative abundance for some taxa increased after PMA treatment (g_Pseudomonas, s_Pseudomonas psychrophila, c_Gammaproteobacteria, s_Pseudomonas fragi, ${ }_{S}$ Pseudomonas koreensis, $k_{-}$Bacteria), while all taxa showed a decrease in absolute abundance. This indicates that PMA treatment may increase the ability to detect taxa with majoritarily viable populations. We did not detect new taxa that were previously undetectable in PMA-treated samples. However, if nonviable microbes are not of interest, treating samples with PMA will improve the detection power for the overall community with comparable sequencing resources (Fig. 6c), as it reduced the overall $\boldsymbol{\alpha}$ diversity (Fig. 3a).

Cross referencing between cultivation and PMA-MetaSeq was greatly impeded by their inherent limitations (e.g., detection limit for MetaSeq, biases with bioinformatics; viable but non-culturable cells for cultivation). Even for PMA-untreated samples, cultivation and MetaSeq only agreed with each other on a small number of taxa (Fig. 4a). Among the 3 viable taxa confirmed by cultivation, viability of $s_{-}$Pseudomonas fragi and $s_{-}$Pseudomonas stutzeri was reflected by PMA-MetaSeq, while $S_{-}$Pseudomonas fluorescens became undetectable after PMA treatment. This might imply over-depletion, but could also be because its abundance went below the detection limit of MetaSeq. As indicated by Barbau-Piednoir et al., less-abundant taxa were more likely to be eliminated (to undetectable) by PMA treatment ${ }^{44}$. This is consistent with our observation, as the abundance of s_Pseudomonas fluorescens was the smallest among the cultivation-confirmed taxa. Thus for lowabundance taxa, cultivation could serve as a supplement to sequence-based viability assessment techniques, as a small unintentional removal of viable cells may lead to a large presence/absence difference. Moreover, incorporating cultivation can expand the detection spectrum in general, and particularly for low-abundance taxa, due to MetaSeq's restrictions such as detection limit and failure to distinguish closely related taxa.

Collectively, we emphasize the importance of using absolute abundance and demonstrate a successful application of multi-species internal standards in PMA-MetaSeq. PMA is effective in low biomass samples and can improve the detection power by eliminating irrelevant signals. Cultivation remains a valuable supplement to sequence-based techniques for capturing a comprehensive picture. 

metagenomics

190 Quantifying metagenomics-based abundances using internal standards has substantial benefits. 191 Theoretically, addition of internal standards could compensate for errors resulting from nonquantitative steps ${ }^{13}$. E. coli is one of the most used spike-in strains, in part because it is well-studied and easy to recover in sequencing ${ }^{23}$. However, ideally, we want the internal standard to contain a set of diverse taxa, so that it well represents the diversity in microbial communities. We investigated the performance of the Zymo community as the internal standard for hospitalassociated environmental samples, along with qPCR for the 16S rRNA gene.

197 Unfortunately, the efficiency of implementing the Zymo standard in quantitative metagenomics 198 was drastically impeded by the limited resolution of taxonomic classification. We tried two approaches: Metaxa2 ${ }^{45-47}$ coupled with the SILVA 132 SSU database ${ }^{48,49}$ and MetaPhlAn3 ${ }^{50,51}$, which uses a collection of marker genes. The taxonomic resolution varied substantially across different taxa. For samples containing only the Zymo standard, $85 \%$ of the small subunit rRNA reads were attributable by Metaxa2, while only $48.14 \%$ of the metagenomes were recognized by MetaPhlAn3. Within the attributable portion, MetaPhlAn3 performed better regarding specificity; all reads were classified at the species level, while Metaxa2 retained a decent amount of information at higher levels, with the ratio of genus/family-level and species-level classifications ranging from 0.18 to 11.28 .

Foreseeably, this issue will be alleviated as reference databases and taxonomic assignment tools continue to advance. Currently, advantages of internal standards are mainly reflected when species-level identification is the major focus. For instance, clinical samples usually target pathogenic species whose core pangenomes are relatively well represented in databases. In this case, the biases from uneven representation of species can also be corrected based on the performance of closely related internal-standard species. However, if information at genus or higher levels is of consideration, internal-standard techniques become non-applicable, as we are not able to distinguish internal-standard taxa from other species within the same genus (or at higher levels), which is the basis of making calculations and corrections. Coupling with qPCR, instead, is more appropriate (Fig. 3c). Environmental communities are typical examples where coupling with qPCR stands out because environmental microorganisms are not usually well represented at the species level. Of 87 samples in our study, strikingly, MetaPhlAn3 only recognized an average of $19.68 \%$ of the metagenome at the species level. The classification rate slightly increased to $38.40 \%$ using Metaxa2, which substantially improved to $87.24 \%$ when genus level was included.

\section{Accessible sample features can predict required sequencing effort}

To enable more informed decision-making before MetaSeq, we conducted a quasi-meta-analysis, using the limited number of existing hospital-related environmental metagenomics studies ${ }^{1,5,20,52-}$ 54 . We recruited 956 shotgun samples ( 874 from 6 previous studies and 82 from this study) (Supplementary Table 1). Using these data, we linked accessible features (e.g., location, building, sampling method) to the required sequencing effort given a targeted coverage, leveraging machine learning-based models and Nonpareil (Fig. 6a). 
Relationships between Nonpareil diversity (Nd, unit: log-bp) and metadata features were first explored (Fig. 6a: stage one). $\mathrm{Nd}$ is an index measuring the complexity of a microbial community regarding "sequence space", which correlates with classic bin-based diversity indices (e.g., Shannon index) for bacteria ${ }^{35,37}$. Though not passing the normality test (Shapiro-Wilk Test, $\mathrm{p}=$ $3.338 \mathrm{e}-16)^{55}$, normal distribution was still the best-fit distribution of our dataset, followed by logistic distribution, upon investigation by Cullen and Frey Graph and R package "fitdistrplus" (Fig. 5a, Supplementary Fig. 7). Presumably, the deviation from normality will decrease as sample sizes increase. For $90 \%$ of samples, $\mathrm{Nd}$ was within 2 orders of magnitude (15.4-20.0, natural log scale), suggesting a common range for hospital-associated environmental samples, which is valuable for reference when designing future studies. Notably, this $\mathrm{Nd}$ level was among the lowest across 6 different environments including animal hosts, fresh water, and soil (Fig. 5b $)^{37}$.

We further examined the influences of sample type (sink versus surface), sampling method and sample pooling on $\mathrm{Nd}$. No significant difference was observed between sink and surface samples (Fig. 5c). Within sink samples, $\mathrm{Nd}$ was significantly different across sampling methods (Anova, $\mathrm{p}$ $=7.18 \mathrm{e}-06$ ). Specifically, samples collected by swabs seemed to have a smaller diversity than those by the other methods (Tukey's post-hoc test; samples without a clear collection method stated in the original paper were assigned as "Sink"). Note that even though sink samples are generally from the same location, the confounding effects introduced by sub-locations (e.g., sink basin, pipe edge, $p$ trap) cannot be ruled out. Similarly, within surface samples, though Nd of wipes was significantly larger than that of swabs (unpaired t-test), confounding effects remain (e.g., researchers tend to use wipes for large-area and high-biomass locations, like floors, which often contain more diverse communities) (Fig. 5d). Though weak, we noticed a trend of diversity increase after sample pooling (Fig. 5e), raising the alarm that more caution should be taken when increasing biomass by pooling samples. The practice of sample pooling assumes that the pooled samples share some core features, whose biomass will be increased past the detection limit. This may be true of certain sample types, e.g., host-associated microbiomes, but is unlikely to be true of built-environment samples that lack a conserved core ${ }^{56}$. Further investigations are needed should more data become available, as the sample size was quite limited for some groups (e.g., $\mathrm{n}($ pooled monitor $)=2$, $\mathrm{n}($ not-pooled monitor $)=4)$. In the interim, we recommend seeking other methods, such as a high-yield DNA extraction, before resorting to sample pooling, as the resulting sample characteristics may be different from individual samples.

To further harness the reference potential of $\mathrm{Nd}$, we built models to predict $\mathrm{Nd}$ from metadata features based on machine learning algorithms. Eight predictor variables (location, building, study, country, touch frequency, sample type, sampling method, sample pooling) were included based on data availability, MIxS-BE standards, and previous experience (Supplementary Table 1) ${ }^{53,57,58}$. $\mathrm{Nd}$, the response variable, was first converted from a numerical variable to a nominal variable. Three conversion schemes were tried, with the intervals being 2.5, 1.0, and 0.5 (number of categories being 2, 5, 11, respectively). Random sampling was adopted to split the entire dataset into training and testing datasets at the ratio of 4:1. Implementing repeated cross-validation (5 folds, 5 times) on the training dataset, 9 algorithms were examined to optimize the classification performance, including random forest, stochastic gradient boosting, and support vector machines. Algorithms were evaluated according to 4 metrics (area under curve, Kappa, and balanced 
accuracy on both training and testing datasets $)^{59}$. Overall, no difference was observed among the tested algorithms. Random forest was selected due to its slightly better performance from a holistic perspective and capability of ranking the predictor variables.

273 The model accuracy positively correlated with the interval size. At 2.5, the accuracy on the training dataset was as high as $87.69 \%$, and slightly lower on the testing dataset $(82.60 \%)$. The accuracy dropped as the classification demand rose. The mean balanced accuracy on the testing dataset was $64.08 \%$ and $61.38 \%$ when intervals were 1.0 and 0.5 , respectively. Considering that $\mathrm{Nd}$ was converted from a continuous variable, we examined the misclassifications and found that most of them fell into nearby categories. We thus calculated the mean balanced accuracy \pm 1 category and observed a substantial improvement. Specifically, mean balanced accuracy of $87.06 \%$ and $77.17 \%$ can be achieved for 5- and 11-category classifications, respectively. Considering the sparsity of the currently available dataset and the challenge of multiclass classification, this model demonstrated a reasonable degree of accuracy, which should improve as sample sizes and available features grow.

The variable importance ranking generated by random forest separated the predictor variables into 3 groups (Fig. 6b). Location, building, and study were the top 3 variables with the highest importance, followed by country, touch frequency, and sample type, while sample pooling and sampling method hardly impacted the classification. Group-wise, this ranking was generally consistent with the explanatory power described by linear regression (Supplementary Fig. 8) ${ }^{58}$. That "study" ranked as one of the most important variables indicated the existence of biases towards individual studies in the current dataset (e.g., "batch effects" related to respective sampling, processing, sequencing, and analysis), which was also observed by a previous metaanalysis of indoor microbiota ${ }^{58}$. Interestingly, despite its high importance, the model performance had almost no drop after excluding "study" ( $>95 \%$ for all conversion schemes), justifying making predictions without involving artificial metadata features like "study". It is worth noting that importance of the other variables (e.g., building, country) was raised after this exclusion (Supplementary Fig. 9). To find the features necessary for making a comparably accurate prediction, we further examined the performance of models after gradually reducing the number of predictor variables, and found that using only 2, "location" and "building", the new model achieved $95 \%$ accuracy regardless of interval sizes tested.

With $\mathrm{Nd}$ and metadata features connected, the required sequencing effort at a targeted coverage was then inferred (Fig. 6a: stage two). Upon fitting the data, we revealed a linear relationship between the natural $\log$ of estimated sequencing effort at $95 \%$ coverage (ln(LRstar)) and $\mathrm{Nd}$, with the equation being $\ln (\mathrm{LRstar})=1.14 * \mathrm{Nd}+1.21$ (Adjusted R-squared $=0.6012, \mathrm{p}<2.2 \mathrm{e}-16)($ Fig. 6c). This is theoretically backed up by previous findings that sequencing effort depends on the diversity level and the genome size, and that the latter can be ignored for most microbial communities, particularly bacterial communities, since the differences in genome size are usually no more than one order of magnitude ${ }^{35}$. Instructions to make calculations between sequencing effort and other coverage levels are provided at https://github.com/jxshen311/workflow_metagenomic_environmental_surveillance/tree/main/no npareil/example_SeqEffort\%26Coverage. 


\section{Discussion}

312 Although sequence-based environmental surveillance of microbial communities for better 313 management of public health has been appealed for and utilized, best practices of the workflow 314 have not been systematically studied to ensure proper interpretations of sequencing results to aid 315 in infection risk assessment ${ }^{13,14}$. This study introduces a well-structured and informed 316 metagenomics-based workflow towards the goal of being appropriate for low-biomass, viability, quantification, and resource estimation. We recommend adopting liquid-liquid extraction to improve DNA yield and only incorporating whole-cell filtration when non-bacterial proportion is large. Despite its imperfection, we suggest including PMA treatment, and involving cultivation when demanding comprehensive profiling. We further recommend integrating internal standards for quantification, and additionally qPCR when we expect poor taxonomic classification. We also introduce a machine learning-based model to predict required sequencing effort from accessible sample features. The model helps make full use of sequencing resources and achieve desired outcomes.

While using realistic samples in testing simulates conditions the workflow may face in practical applications, it comes with side effects. Our aggregation sample had a small fraction of nonbacterial organisms $(\sim 1 \%)$. Thus, the conclusion that whole-cell filtration does not increase the bacterial proportion and signal of rare taxa to a statistically significant degree is probably only applicable to samples with similar characteristics, representing $84.90 \%$ among the 874 samples from hospital-related environmental studies used in the quasi-meta-analysis ${ }^{1,5,20,52-54}$. However, a few samples did contain a decent proportion of eukaryotes. Specifically, 132 samples harbored more than $1 \%$ eukaryotic reads, and strikingly more than half reads were attributed to eukaryotes for 20 samples. Moreover, samples collected from high-touch surfaces were more likely to have higher proportions of eukaryotes than low-touch surfaces and sinks. Of the 104 sink samples, the maximum percentage of eukaryotes was $0.1 \%$. Therefore, filtration is probably unnecessary for most environmental samples (especially sink samples) and may be beneficial for part of high-touch surface samples (Supplementary Fig. 10).

Despite being semi-quantitative and entailing considerable uncertainty, involving PMA takes us a step closer to understanding viability, particularly for low biomass samples whose complexity is also relatively $l_{0}{ }^{23}$. Notably, the overall uncertainty comes not only from PMA treatment but also from the metagenomics pipeline itself, such as biases from DNA extraction kits and taxonomic assignment tools ${ }^{16,60}$. Integration with other omics techniques (e.g., metatranscriptomics, metaproteomics) was proposed to make up for PMA's shortcomings ${ }^{23}$. Pursuing viability profiles using orthogonal methods would plausibly enable a more comprehensive understanding, but the cost-benefit ratio may be considerably high for multi-omic techniques. Integrating with cultivation, instead, provides an affordable alternative. Notwithstanding, it remains to be investigated how to properly interpret results generated by a combination of methods, as inconsistencies between disparate methods are common.

Some overlap exists between methods for viability determination and those for depleting eukaryotic DNA. For example, osmotic lysis followed by PMA treatment is recommended to remove human DNA in saliva samples ${ }^{61}$. However, recommended methods depend on the sample 
type. PMA is not recommended for sputum samples, where nuclease-based methods (e.g., digest with benzonase) showed an equal or better performance ${ }^{62}$. Factors impacting method performance include percentage and composition (e.g., extracellular DNA, DNA in largely lysed or partially compromised cells) of targets to be removed (i.e., eukaryotes and dead bacteria), as well as characteristics of background matrix (e.g., viscosity). For instance, saliva and sputum consistently contain $\gtrsim 90 \%$ human $\mathrm{DNA}^{61,62}$, while this percentage is very diverse for hospital-associated environmental samples (Supplementary Fig. 10). Filtration failed to exclude human DNA in saliva likely because extracellular DNA was the dominant component rather than cells ${ }^{61}$. For sputum samples where cells are lysed and DNA is no longer protected, nucleases might be quite effective in depleting extracellular DNA, whereas PMA efficacy could be hindered by the viscosity of the matrix $)^{62}$. In contrast, in environments where cells gradually decay due to harsh conditions (e.g., desiccation), more DNA attributable to dead cells would still have a partially compromised membrane; PMA, as a small molecule, may be more effective in penetrating the damaged cell membrane and depleting the DNA. For eukaryotic depletion, it may be beneficial to further unravel the underlying mechanisms influencing the efficacy of different methods in different sample types and characteristics. For viability assessment, instead of focusing on this viable/dead dichotomy, perhaps more critically, we should keep in mind that "viability" is rather an intermediate or methodological term, linking surveillance results to questions of interest (e.g., which bacteria are infectious) ${ }^{13}$.

We applied multi-taxa internal standards and calculated PMA efficacy of spike-in taxa based on a reasonable assumption that the percentage of viable microbes in the Zymo community is negligible, resulting in a theoretical value of 1 . While this internal standard can strongly reflect incomplete suppression of non-viable signals, potential toxicity of PMA might be underrepresented. Although no toxicity was observed at the PMA dose of our protocol in validation (Supplementary Fig. 11), a customized internal-standard mixture featuring 0.5 as the designed PMA efficacy would be ideal for future studies ${ }^{23}$. As opposed to purchasing commercial products, we recommend utilizing the Zymo community as a reference for the taxonomic composition and constructing the mixture with live cultures in real-time, because viability (or membrane integrity when PMA is used) is difficult to maintain in manufacturing, shipping, and storage.

Continuous advancement of internal standards for quality control, as well as quantification and other features, is still one of the major hotspots in method optimization. A suitable internal standard should well balance representation and recognizability. Good representation means that the workflow impacts the spike-in and targeted microbes comparably (because of their similarity). Good recognizability means that the spike-in can be easily distinguished from the targets. In this study, the Zymo community was selected due in large part to its representation, as it spans broadly the phylogenetic tree. Previous studies have selected internal standards based on a similar principle. For instance, the Zymo community and a 10 -species mock community were chosen for gastrointestinal and stool samples, respectively ${ }^{63,64}$. Peroxide-killed Campylobacter sputorum was used to quantify viable thermotolerant Campylobacter ${ }^{65}$. These internal standards are prone to be confounded with targets, thus posing challenges for bioinformatics to accurately identify and quantify taxa. To obtain good recognizability, exotic materials are sought. In the aforementioned example, the researchers chose 10 species that were generally absent from the stool of healthy 
individuals. The same criterion was followed by another gut microbiome study in which microbes from hypersaline environments, soil, and plants were utilized ${ }^{34}$, as well as a study on Amazon River plume to which genomic DNA from Thermus thermophilus HB8 was applied ${ }^{32}$. Finding a completely exotic species is more challenging for environmental surveillance whose subjects are influenced by both human and environmental activities. As a potential solution, artificial DNA have been developed to ensure differentiation from the targets. Previous reports included sets of synthetic DNA, 16S rRNA genes, and chimeric DNA fragments, implemented in different venues of metagenomic and amplicon sequencing ${ }^{33,66,67}$. However, whether these exogenous (or even artificial) standards' behavior resembles that of the targets remains questionable. By and large, more systematic evaluation and optimization are needed to foster the development of internalstandard techniques that better balance representation and recognizability, or at least make their pros and cons quantitatively accessible, both in general and for specific contexts.

Though the classification models performed well from a practical perspective, their accuracy with small intervals still merits improvement. Building a hierarchical classification model might be beneficial, as we observed a drastic increase in the accuracy when the interval size was enlarged. It is also likely that the available dataset is not good enough to train a model with very high accuracy. For example, there is clear evidence that the data were biased by the disparate sample sizes between studies. Moreover, we only managed to collect 7 common metadata features (excluding "study") without involving a substantial number of missing values, which raises the question of whether what we achieved has already reached the theoretical plateau of explanatory power of these features. If this is the case, standardized reporting of more high-quality metadata should be further promoted. Additionally, since normal distribution was the best-fit distribution of the current dataset, with seemingly missing pieces in the middle (Fig. 5a), fitting data into known distributions may be more explanatory as large sizes of data become accessible.

This metagenomics-based environmental surveillance workflow is particularly useful in infection prevention and disinfection assessment. Although we focus on microbial surveillance of built environments, especially hospital-associated surfaces, the workflow developed in this study can be adapted to other contexts with similar characteristics. For example, the multifaceted lessons learned from this study will benefit the continuing development of microbiome-based clinical testings from body sites (e.g., skin), such as methods to increase low-biomass signals and determine viability ${ }^{12}$. Moreover, the experience gained in overcoming challenges unique to environmental microbiomes (e.g., quantitative metagenomics with poor taxonomic classifications) is also useful to studies on other environments, such as wastewater and air.

\section{Methods}

\section{Sample collection, aggregation, and cultivation}

We collected 120 surface swabs from the 28-bed medical intensive care unit (MICU) at Rush University Medical Center (RUMC) in October 2018. RUMC is a 720-bed tertiary care teaching hospital in Chicago, IL. Samples were collected from door sills, computer keyboards, light switches, nurse call buttons, and bed rails in 13 single-bed patient rooms, as well as door sills in 4 
medication rooms, 2 public restrooms, 1 staff-only restroom, and the communicating space of MICU (Supplementary Table 2). Weighted mean area of sampled surfaces was $216 \mathrm{~cm}^{2}$. Patient rooms were selected to keep a relatively balanced number for both contact isolation and noncontact isolation rooms. Healthcare providers and visitors entering contact isolation rooms are required to wear gowns and gloves, which may reduce transmissions via contaminated healthcare providers. Room temperature and relative humidity were documented during the collection, which varied slightly across rooms, with the average being $23.8^{\circ} \mathrm{C}$ and $45 \%$, respectively. Each sample was collected by 3 COPAN Nylon Flocked Swabs (Copan Diagnostics, Murrieta, CA, USA) and $1.5 \mathrm{~mL}$ Phosphate Buffered Saline with $0.02 \%$ Tween 80 (PBST), and stored at $4^{\circ} \mathrm{C}$ for up to 24 $\mathrm{h}$ prior to extraction, aggregation, and cultivation ${ }^{53,68}$. Swabs were extracted and aggregated to create a representative microbiome sample ${ }^{56,68,69}$. Aliquots of this aggregation sample were then subjected to different processing methods (i.e., several DNA extraction methods, microbial community standard spike-in, PMA treatment and whole-cell filtration) to find best practices of the workflow (Supplementary Fig. 1).

447 To capture a large fraction of the indoor microbiome diversity, we cultured the samples with 4 different media: tryptic soy agar (TSA), Reasoner's $2 \mathrm{~A}$ agar (R2A), 0.1 strength R2A at $25^{\circ} \mathrm{C}$, and blood agar (BA) at $37^{\circ} \mathrm{C}$, all supplemented with $4 \mathrm{mg} / \mathrm{L}$ itraconazole ${ }^{68}$. This resulted in 233 cultivable isolates. All colonies that could be individually picked or purified were subject to taxonomic identification by matrix-assisted laser desorption/ionization time-of-flight mass spectrometry (MALDI-TOF MS) using the VITEK ${ }^{\circledR}$ MS Mass spectrometry microbial identification system (BioMerieux, Marcy-l'Étoile, France) and the VITEK MS V3.2 FDA 510(k) cleared database. Among the 233 isolates, 201 were identified. It is important to note that, because multiple media types were used, the number of isolates for each species identified does not represent the relative abundance of this species in the sample, as some species may have grown on multiple media.

\section{Standard addition, PMA treatment and whole-cell filtration}

459 All treatments were done in triplicate, including cultivation.

460 Standard addition

461 Aliquots were snap frozen and stored at $-80^{\circ} \mathrm{C}$ until further processing to maximize the integrity 462 of samples and avoid degradation resulting from long-term storage at $4^{\circ} \mathrm{C}^{69,70}$. Samples were 463 thawed at $4^{\circ} \mathrm{C}$ prior to treatments. ZymoBIOMICS Microbial Community Standard (Zymo 464 Research, Irvine, CA, USA) was used as both the internal standard and the external standard. As the internal standard, $6.50 \mu \mathrm{L}$ Zymo community was spiked into $1 \mathrm{~mL}$ aggregate sample, following the criterion that DNA of the species with the highest abundance in the Zymo community approximates $1 \%$ of the total DNA in the aggregate sample ${ }^{32,71}$. As the external standard, aliquots of the Zymo community were run in parallel with aggregate samples throughout the workflow to assure its performance. 
PMA treatment

471 Following standard addition, PMA treatment (Biotium, Fremont, CA, USA) with an optimized protocol was applied to half of the samples within each group $22,24,25,72,73$. The protocol was first

\section{Whole-cell filtration} validated by reproducing the work of Nocker et al. (2006) using Escherichia coli (ATCC 8739) as model strain. E. coli was grown to the exponential phase and half killed by heat inactivation at $95^{\circ} \mathrm{C}$ for $7 \mathrm{~min}$ in Eppendorf ThermoMixer shaking at $400 \mathrm{rpm}$ for homogenized heating. After cooling to room temperature, live and heat-killed cultures were mixed following the same ratios as in Nocker et al. (2006), yielding samples of 6 different expected live cell ratios. Viability of both live and heat-killed cultures was confirmed by spread plating onto TSA and incubating at $37^{\circ} \mathrm{C}$ overnight. Half of the constructed samples underwent PMA treatment. The results were evaluated by both DNA concentration ratio quantified by Quant-iT ${ }^{\mathrm{TM}}$ PicoGreen ${ }^{\mathrm{TM}}$ dsDNA Assay (ThermoFisher, Waltham, MA, USA) and copy number ratio by qPCR with $16 \mathrm{~S}$ universal primers (341F and 534R) (Supplementary Fig. 11). Briefly, a final concentration of $25 \mu \mathrm{M}$ PMA was used, and several steps were conducted to ensure the consistency across samples and minimize nonspecific reactions between PMA and random sample components, including 1) adding PMA to tube caps and inverting all tubes simultaneously, 2) working under red light, and 3) protecting samples from light as much as possible before the light activation step in the PMA-Lite ${ }^{\mathrm{TM}}$ device (Biotium, Fremont, CA, USA). An aliquot of samples for each replicate was preserved at $-80^{\circ} \mathrm{C}$ until DNA extraction, with the rest stored at $4^{\circ} \mathrm{C}$ for downstream filtration.

Whole-cell filtration was conducted using EMD Millipore $25 \mathrm{~mm}$ Glass Vacuum Filter kit (MilliporeSigma, Burlington, MA, USA), $125 \mathrm{~mL}$ filter flask, and Gemini vacuum pump in a biosafety cabinet following aseptic techniques (Supplementary Fig. 12). Notably, autoclaved tweezers were used to avoid possible contaminations from touching sensitive parts of the set-up. Samples were filtered by $100 \mu \mathrm{m}$ nylon membrane, followed by $80 \mu \mathrm{m}$ and $41 \mu \mathrm{m}$ nylon membranes and $5 \mu \mathrm{m}$ PVDF membrane (MilliporeSigma, Burlington, MA, USA). $1 \mathrm{~mL}$ PBS was added to the falcon tube and flask at each step to increase the sample recovery by rinsing the inner wall. The filtered samples were then subjected to 3-fold (relative to the volume before filtration) vacuum concentration with an Eppendorf Vacufuge plus. Filtered liquid samples and filter papers were preserved at $-80^{\circ} \mathrm{C}$ until DNA extraction. To increase the extraction efficiency from filter papers, we compared 1) cutting them with scissors into 9 pieces, 2) grinding them with metal spatula after snap freezing in liquid nitrogen, and 3) directly putting the whole filter paper into the preservation tube. We finally selected the third option as this was the most operationally feasible way without high risk of contamination.

\section{Negative controls}

505 To combat the susceptibility of low-biomass samples to contamination, we included 4 types of negative controls along the workflow, namely, 6 negative field controls, 6 negative media controls, 12 negative filter controls, and 7 negative kit controls ${ }^{13,15,16,58}$. The negative controls were 508 processed in parallel with the surface samples, including metagenomic sequencing and 509 bioinformatic analysis. 
Negative field controls were collected exactly the same as surface samples, except that the swabs were exposed to the air without contacting targeted surfaces. One negative field control was collected at the beginning and the end of each sampling session. Two negative media controls were included in each sampling session, which were unopened media with swabs from the same lot. Each collected control was split into triplicate and processed along with samples ${ }^{53,74}$. Negative filter controls were included in triplicate for each pore size by letting sterile PBST flow through the vacuum filtration system attached with blank filter papers. Additionally, 7 negative kit controls were processed across batches of DNA extractions.

\section{DNA extraction, qPCR and metagenomic sequencing}

519 To ensure enough DNA recovery, we performed an initial optimization on a separate set of surface swab samples collected from the same MICU prior to working with the aggregate sample (Supplementary Fig. 2). Column-based methods were first tried due to its widespread usage in the field. We examined Qiagen DNeasy PowerSoil Kit with standard protocol and a modified version by 1) changing from vortex lysis to bead-beating lysis, 2) introducing heat incubation after bead-beating, and 3) adding $50 \mu \mathrm{L}$ water each time for twice in total at the elution step. DNA yields of both were below the limit of detection. Liquid-liquid extractions were performed afterwards for their high-yielding potentials. Phenol-chloroform extraction resulted in the highest yield (186.27 $\pm 55.51 \mathrm{ng} / \mu \mathrm{L}$ by NanoDrop), but the purity indicated by $260 / 280$ was not acceptable (1.36 \pm 0.03$)$. Lucigen MasterPure ${ }^{\mathrm{TM}}$ Complete DNA and RNA Purification Kit also resulted in high yields when coupled with bead-beating and heat lysis and better purity than phenolchloroform extraction (260/280 1.62 \pm 0.02$)$. We attempted to improve the purity using the Agencourt AMPure XP PCR Purification kit. However, we did not see a purity increase (260/280 $1.62 \pm 0.03)$ and incurred a $64.70 \%$ DNA yield drop. Based on the above tests, we noticed that methods involving columns (Qiagen PowerSoil) or magnetic beads (Agencourt AMPure) greatly decreased the DNA yield. Because the primary concern for surface samples is low biomass, increasing DNA yield is considered more critical than bringing 260/280 to the desired range of 1.8-2.0. Therefore, the Lucigen MasterPure ${ }^{\mathrm{TM}}$ Complete DNA and RNA Purification Kit with the adapted protocol was chosen for all subsequent analyses ${ }^{75}$. Samples were thawed at $4{ }^{\circ} \mathrm{C}$ prior to DNA extraction and DNA concentrations were quantified by Quant-iT ${ }^{\mathrm{TM}}$ PicoGreen $^{\mathrm{TM}}$ dsDNA Assay ${ }^{76}$.

V3 region of the 16S rRNA gene was amplified in qPCR using universal primers (341F: 5'-CCT ACG GGA GGC AGC AG-3', 543R: 5'-ATT ACC GCG GCT GCT GGC A-3') $)^{40}$. The $20 \mu \mathrm{L}$ reaction mixture consisted of $10 \mu \mathrm{L}$ PowerUp ${ }^{\mathrm{TM}}$ SYBR ${ }^{\mathrm{TM}}$ Green Master Mix (Applied Biosystems), $0.6 \mu \mathrm{L}$ forward primer $(10 \mu \mathrm{M}), 0.6 \mu \mathrm{L}$ reverse primer $(10 \mu \mathrm{M}), 5.0 \mu \mathrm{L}$ DNA templates (pre-diluted if necessary), and $3.8 \mu \mathrm{L}$ nuclease-free water. The reaction was run in technical triplicate on a QuantStudio 3 Real-Time PCR System (Applied Biosystems) with an initial denaturation step at $95^{\circ} \mathrm{C}$ for $2 \mathrm{~min}$, followed by 40 amplification cycles $\left(95^{\circ} \mathrm{C}, 15 \mathrm{~s}\right.$; $\left.56^{\circ} \mathrm{C}, 15 \mathrm{~s} ; 72^{\circ} \mathrm{C}, 1 \mathrm{~min}\right)$ and a melting curve stage $\left(95^{\circ} \mathrm{C}, 15 \mathrm{~s} ; 60^{\circ} \mathrm{C}, 1 \mathrm{~min} ; 95^{\circ} \mathrm{C}, 15 \mathrm{~s}\right)$. Notemplate control and 5 8 standards were included in each batch to generate the standard curve (efficiency $>90 \%$; $\mathrm{R}^{2}>0.99$ ). Plasmid DNA constructed by TOPO ${ }^{\mathrm{TM}}$ TA Cloning ${ }^{\mathrm{TM}} \mathrm{Kit}$ (Invitrogen, Waltham, MA, USA) was used as standards. 
Extracted DNA was shipped on dry ice to the UMICH Microbiome Core (Ann Arbor, MI, USA) for library preparation using Nextera ${ }^{\mathrm{TM}}$ DNA Flex Library Prep Kit and paired-end 250-bp shotgun metagenomic sequencing on an Illumina MiSeq platform (MiSeq Reagent Kit v2). Libraries were normalized before sequencing, and for samples without enough DNA (e.g., negative controls), all available materials were used.

\section{Data analysis}

\section{Sequence data processing}

KneadData (v0.6.1) was first used to clean the shotgun sequences with default parameters. Reads present in the human reference database (hg37_and_human_contamination) and negative controls were filtered out. Metaxa2 (v2.2) (5-47 $^{45}$ coupled with SILVA 132 SSU database ${ }^{49,77}$ was chosen to generate taxonomic profiles after comparing it with MetaPhlAn2 (v2.6.0 $)^{78}$ and MetaPhlAn3 (v3.0.7) ${ }^{50,51}$. The evaluation was conducted based on their performance on external standards and cross validation with cultivation results for untreated aggregate samples. Default parameters were used for all three tools. MetaPhlAn3 was ruled out mainly because it only generates marker genes at the species level and an average of $80.32 \%$ metagenome was deemed unknown for our samples. For external standards, both Metaxa2 and MetaPhlAn2 recognized all 8 bacteria species demonstrated in the theoretical composition, but MetaPhlAn2 failed to classify the 2 eukaryotic species. Moreover, it did not classify Pseudomonas fluorescens and barely classified Pseudomonas stutzeri from aggregate samples, while Metaxa2 recognized both. Though Metaxa2 included a few spurious taxa, all can be eliminated by removing singletons. Since the primary goal of this study was to compare techniques and recommend best practices, sensitivity outweighed specificity. Therefore, Metaxa2 was selected and singletons were removed for downstream analyses. Taxa were labeled to the lowest classifiable level, with species level as the ultimate target ${ }^{23}$. Metagenomic sequencing coverage for all samples was estimated by Nonpareil (v3.303) under kmer mode using default settings ${ }^{35,37}$.

\section{$\underline{\text { Statistical analysis }}$}

Statistical analyses and data visualization were conducted in R (v4.0.4) ${ }^{79}$ with packages such as Nonpareil, vegan, ape, ggplot ${ }^{80}$, and dplyr. Principal coordinate analysis (PCoA) based on Jaccard metric was performed to demonstrate beta diversity ${ }^{58}$. Differences between groups were determined by Student's T-test or ANOVA coupled with Tukey's post-hoc test, depending on the number of groups under comparison. $\mathrm{P} \leq 0.05$ was defined as statistically significant.

Significance codes are: $\mathrm{p}>0.05$ (ns), $0.01<\mathrm{p} \leq 0.05\left(^{*}\right), 0.001<\mathrm{p} \leq 0.01(* *), \mathrm{p} \leq 0.001\left(^{* * *}\right)$. Package "fitdistrplus" was implemented to find the best-fit distribution for Nonpareil diversity. Machine learning models were trained using the package "caret".

\section{Data availability}

The raw shotgun metagenomic sequencing data are available in the NCBI SRA repository under Bioproject number PRJNA765404. Source code and supplementary data for reproducing analyses 
are available

under

MIT

license

at

589 https://github.com/jxshen311/workflow metagenomic environmental surveillance. Protocols are 590 available at Protocol Exchange with DOIs: 10.21203/rs.3.pex-1656/v1 (sample collection, 591 extraction, and cultivation), 10.21203/rs.3.pex-1657/v1 (snap freezing), 10.21203/rs.3.pex592 1659/v1 (PMA treatment), and 10.21203/rs.3.pex-1658/v1 (DNA extraction).

\section{3}

594

595

596

597

598

599

600

601

602

603

604

605

606

607

608

609

610

611

612

613

614

615

616

617

618

6195

620

621

622

623

\section{Acknowledgments}

This work was supported by the Centers for Disease Control and Prevention (BAA FY2018OADS-01 Contract 02915). This research was supported in part through the computational resources and staff contributions provided for the Quest high performance computing facility at Northwestern University which is jointly supported by the Office of the Provost, the Office for Research, and Northwestern University Information Technology. We thank Thelma Dangana and Khaled Aboushaala for their help in collecting samples, and the clinical staff of the medical intensive care unit at Rush University Medical Center for their cooperation. We are also grateful to Pamela B Bell and Rachel Beers for their contributions in performing MALDI-TOF MS.

\section{Author's contributions}

JS and EMH designed the study. JS conducted the experiments, performed the analyses, and wrote the manuscript. JS and AM collected the samples. AM and RAB provided support in bioinformatics. LJR, AP, MKH, VBY, and EMH supervised the project. All authors have read, edited, and approved of the final manuscript.

\section{References}

1 Brooks, B. et al. Strain-resolved analysis of hospital rooms and infants reveals overlap between the human and room microbiome. Nature Communications 8, 1814, doi:10.1038/s41467-017-02018-w (2017).

2 Raveh-Sadka, T. et al. Evidence for persistent and shared bacterial strains against a background of largely unique gut colonization in hospitalized premature infants. Isme $j \mathbf{1 0}$, 2817-2830, doi:10.1038/ismej.2016.83 (2016).

3 Lax, S. et al. Longitudinal analysis of microbial interaction between humans and the indoor environment. Science 345, 1048, doi:10.1126/science.1254529 (2014).

$4 \quad$ Vickery, K. et al. Presence of biofilm containing viable multiresistant organisms despite terminal cleaning on clinical surfaces in an intensive care unit. Journal of Hospital Infection 80, 52-55, doi:https://doi.org/10.1016/j.jhin.2011.07.007 (2012).

5 Constantinides, B. et al. Genomic surveillance of Escherichia coli and Klebsiella spp. in hospital sink drains and patients. Microb Genom 6, doi:10.1099/mgen.0.000391 (2020).

6 Martineau, C. et al. Serratia marcescens Outbreak in a Neonatal Intensive Care Unit: New Insights from Next-Generation Sequencing Applications. Journal of Clinical Microbiology 56, e00235-00218, doi:doi:10.1128/JCM.00235-18 (2018). 
6247 Hu, H. et al. Intensive care unit environmental surfaces are contaminated by multidrug625 resistant bacteria in biofilms: combined results of conventional culture, pyrosequencing, scanning electron microscopy, and confocal laser microscopy. J Hosp Infect 91, 35-44, doi:10.1016/j.jhin.2015.05.016 (2015).

8 Burgos-Garay, M. et al. Colonization of carbapenem-resistant Klebsiella pneumoniae in a sink-drain model biofilm system. Infect Control Hosp Epidemiol 42, 722-730, doi:10.1017/ice.2020.1287 (2021).

9 Kotay, S., Chai, W., Guilford, W., Barry, K. \& Mathers, A. J. Spread from the Sink to the Patient: In Situ Study Using Green Fluorescent Protein (GFP)-Expressing Escherichia coli To Model Bacterial Dispersion from Hand-Washing Sink-Trap Reservoirs. Applied and environmental microbiology 83, e03327-03316, doi:10.1128/AEM.03327-16 (2017).

10 D'Souza, A. W. et al. Spatiotemporal dynamics of multidrug resistant bacteria on intensive care unit surfaces. Nat Commun 10, 4569, doi:10.1038/s41467-019-12563-1 (2019).

11 Ben Maamar, S. et al. Mobilizable antibiotic resistance genes are present in dust microbial communities. PLoS Pathog 16, e1008211, doi:10.1371/journal.ppat.1008211 (2020).

12 Selway, C. A., Eisenhofer, R. \& Weyrich, L. S. Microbiome applications for pathology: challenges of low microbial biomass samples during diagnostic testing. The Journal of Pathology: Clinical Research 6, 97-106, doi:10.1002/cjp2.151 (2020).

13 Shen, J., McFarland, A. G., Young, V. B., Hayden, M. K. \& Hartmann, E. M. Toward Accurate and Robust Environmental Surveillance Using Metagenomics. Frontiers in Genetics 12, doi:10.3389/fgene.2021.600111 (2021).

14 Nayfach, S. \& Pollard, K. S. Toward Accurate and Quantitative Comparative Metagenomics. Cell 166, 1103-1116, doi:10.1016/j.cell.2016.08.007 (2016).

15 Eisenhofer, R. et al. Contamination in Low Microbial Biomass Microbiome Studies: Issues and Recommendations. Trends in Microbiology 27, 105-117, doi:https://doi.org/10.1016/j.tim.2018.11.003 (2019).

16 McLaren, M. R., Willis, A. D. \& Callahan, B. J. Consistent and correctable bias in metagenomic sequencing experiments. eLife 8, e46923, doi:10.7554/eLife.46923 (2019).

17 Martí, J. M. Recentrifuge: Robust comparative analysis and contamination removal for metagenomics. PLOS Computational Biology 15, e1006967, doi:10.1371/journal.pcbi.1006967 (2019).

18 Davis, N. M., Proctor, D. M., Holmes, S. P., Relman, D. A. \& Callahan, B. J. Simple statistical identification and removal of contaminant sequences in marker-gene and metagenomics data. Microbiome 6, 226, doi:10.1186/s40168-018-0605-2 (2018).

19 Bowers, R. M. et al. Impact of library preparation protocols and template quantity on the metagenomic reconstruction of a mock microbial community. BMC Genomics 16, 856, doi:10.1186/s12864-015-2063-6 (2015).

20 Mahnert, A. et al. Man-made microbial resistances in built environments. Nature Communications 10, 968, doi:10.1038/s41467-019-08864-0 (2019).

21 Minich, J. J. et al. KatharoSeq Enables High-Throughput Microbiome Analysis from LowBiomass Samples. mSystems 3, e00218-00217, doi:10.1128/mSystems.00218-17 (2018).

22 Nocker, A., Cheung, C. Y. \& Camper, A. K. Comparison of propidium monoazide with ethidium monoazide for differentiation of live vs. dead bacteria by selective removal of 
DNA from dead cells. $J$ Microbiol Methods 67, 310-320, doi:10.1016/j.mimet.2006.04.015 (2006).

23 Wang, Y. et al. Whole microbial community viability is not quantitatively reflected by propidium monoazide sequencing approach. Microbiome 9, 17, doi:10.1186/s40168-02000961-3 (2021).

24 Elizaquivel, P., Aznar, R. \& Sanchez, G. Recent developments in the use of viability dyes and quantitative PCR in the food microbiology field. J Appl Microbiol 116, 1-13, doi:10.1111/jam.12365 (2014).

25 Fittipaldi, M., Nocker, A. \& Codony, F. Progress in understanding preferential detection of live cells using viability dyes in combination with DNA amplification. Journal of Microbiological Methods 91, 276-289, doi:https://doi.org/10.1016/j.mimet.2012.08.007 (2012).

26 Morton, J. T. et al. Establishing microbial composition measurement standards with reference frames. Nat Commun 10, 2719, doi:10.1038/s41467-019-10656-5 (2019).

27 Liwinski, T., Leshem, A. \& Elinav, E. Breakthroughs and Bottlenecks in Microbiome Research. Trends Mol Med 27, 298-301, doi:10.1016/j.molmed.2021.01.003 (2021).

28 Vandeputte, D. et al. Quantitative microbiome profiling links gut community variation to microbial load. Nature 551, 507-511, doi:10.1038/nature24460 (2017).

29 Hingamp, P. et al. Exploring nucleo-cytoplasmic large DNA viruses in Tara Oceans microbial metagenomes. ISME J 7, 1678-1695, doi:10.1038/ismej.2013.59 (2013).

30 Satinsky, B. M., Gifford, S. M., Crump, B. C. \& Moran, M. A. in Methods in Enzymology Vol. 531 (ed Edward F. DeLong) 237-250 (Academic Press, 2013).

31 Yamamoto, N., Hospodsky, D., Dannemiller, K. C., Nazaroff, W. W. \& Peccia, J. Indoor emissions as a primary source of airborne allergenic fungal particles in classrooms. Environ Sci Technol 49, 5098-5106, doi:10.1021/es506165z (2015).

32 Satinsky, B. M. et al. The Amazon continuum dataset: quantitative metagenomic and metatranscriptomic inventories of the Amazon River plume, June 2010. Microbiome 2, 17, doi:10.1186/2049-2618-2-17 (2014).

33 Tkacz, A., Hortala, M. \& Poole, P. S. Absolute quantitation of microbiota abundance in environmental samples. Microbiome 6, 110, doi:10.1186/s40168-018-0491-7 (2018).

34 Stammler, F. et al. Adjusting microbiome profiles for differences in microbial load by spike-in bacteria. Microbiome 4, 28, doi:10.1186/s40168-016-0175-0 (2016).

35 Rodriguez, R. L. \& Konstantinidis, K. T. Nonpareil: a redundancy-based approach to assess the level of coverage in metagenomic datasets. Bioinformatics 30, 629-635, doi:10.1093/bioinformatics/btt584 (2014).

36 Rodriguez, R. L. \& Konstantinidis, K. T. Estimating coverage in metagenomic data sets and why it matters. ISME J 8, 2349-2351, doi:10.1038/ismej.2014.76 (2014).

37 Rodriguez-R, L. M., Gunturu, S., Tiedje, J. M., Cole, J. R. \& Konstantinidis, K. T. Nonpareil 3: Fast Estimation of Metagenomic Coverage and Sequence Diversity. mSystems 3, e00039-00018, doi:10.1128/mSystems.00039-18 (2018).

38 Leung, M. H., Wilkins, D., Li, E. K., Kong, F. K. \& Lee, P. K. Indoor-air microbiome in an urban subway network: diversity and dynamics. Appl Environ Microbiol 80, 6760-6770, doi:10.1128/AEM.02244-14 (2014). 
39 Davis, A. et al. Improved yield and accuracy for DNA extraction in microbiome studies with variation in microbial biomass. BioTechniques 66, 285-289, doi:10.2144/btn-20190016 (2019).

$71340 \mathrm{Hu}$, J. et al. Impacts of indoor surface finishes on bacterial viability. Indoor Air 29, 551562, doi:10.1111/ina.12558 (2019).

41 Mo, Y. et al. Biogeographic patterns of abundant and rare bacterioplankton in three subtropical bays resulting from selective and neutral processes. ISME $J \mathbf{1 2}, 2198-2210$, doi:10.1038/s41396-018-0153-6 (2018).

42 Nyirabuhoro, P. et al. Seasonal Variability of Conditionally Rare Taxa in the Water Column Bacterioplankton Community of Subtropical Reservoirs in China. Microbial Ecology 80, 14-26, doi:10.1007/s00248-019-01458-9 (2020).

$43 \mathrm{Ji}, \mathrm{B}$. W. et al. Quantifying spatiotemporal variability and noise in absolute microbiota abundances using replicate sampling. Nature methods 16, 731-736, doi:10.1038/s41592019-0467-y (2019).

44 Barbau-Piednoir, E. et al. Evaluation of viability-qPCR detection system on viable and dead Salmonella serovar Enteritidis. J Microbiol Methods 103, 131-137, doi:10.1016/j.mimet.2014.06.003 (2014).

45 Bengtsson-Palme, J. et al. Metaxa2 Database Builder: enabling taxonomic identification from metagenomic or metabarcoding data using any genetic marker. Bioinformatics 34, 4027-4033, doi:10.1093/bioinformatics/bty482 (2018).

46 Bengtsson-Palme, J., Thorell, K., Wurzbacher, C., Sjöling, Å. \& Nilsson, R. H. Metaxa2 Diversity Tools: Easing microbial community analysis with Metaxa2. Ecological Informatics 33, 45-50, doi:https://doi.org/10.1016/j.ecoinf.2016.04.004 (2016).

47 Bengtsson-Palme, J. et al. METAXA2: improved identification and taxonomic classification of small and large subunit rRNA in metagenomic data. Mol Ecol Resour 15, 1403-1414, doi:10.1111/1755-0998.12399 (2015).

48 Quast, C. et al. The SILVA ribosomal RNA gene database project: improved data processing and web-based tools. Nucleic Acids Res 41, D590-596, doi:10.1093/nar/gks1219 (2013).

49 Yilmaz, P. et al. The SILVA and "All-species Living Tree Project (LTP)" taxonomic frameworks. Nucleic acids research 42, D643-D648, doi:10.1093/nar/gkt1209 (2014).

50 Beghini, F. et al. Integrating taxonomic, functional, and strain-level profiling of diverse microbial communities with bioBakery 3. eLife 10, e65088, doi:10.7554/eLife.65088 (2021).

51 Truong, D. T., Tett, A., Pasolli, E., Huttenhower, C. \& Segata, N. Microbial strain-level population structure and genetic diversity from metagenomes. Genome Res 27, 626-638, doi:10.1101/gr.216242.116 (2017).

52 Lax, S. et al. Bacterial colonization and succession in a newly opened hospital. Sci Transl Med 9, eaah6500, doi:10.1126/scitranslmed.aah6500 (2017).

53 O'Hara, N. B. et al. Metagenomic characterization of ambulances across the USA. Microbiome 5, 125, doi:10.1186/s40168-017-0339-6 (2017).

54 Chng, K. R. et al. Cartography of opportunistic pathogens and antibiotic resistance genes in a tertiary hospital environment. Nat Med 26, 941-951, doi:10.1038/s41591-020-0894-4 (2020). 
55 Mishra, P. et al. Descriptive statistics and normality tests for statistical data. Ann Card Anaesth 22, 67-72, doi:10.4103/aca.ACA_157_18 (2019).

56 Blaustein, R. A. et al. Toothbrush microbiomes feature a meeting ground for human oral and environmental microbiota. Microbiome 9, 32, doi:10.1186/s40168-020-00983-x (2021).

57 Glass, E. M. et al. MIxS-BE: a MIxS extension defining a minimum information standard for sequence data from the built environment. The ISME Journal 8, 1-3, doi:10.1038/ismej.2013.176 (2014).

58 Adams, R. I., Bateman, A. C., Bik, H. M. \& Meadow, J. F. Microbiota of the indoor environment: a meta-analysis. Microbiome 3, 49, doi:10.1186/s40168-015-0108-3 (2015).

59 El Khouli, R. H. et al. Relationship of temporal resolution to diagnostic performance for dynamic contrast enhanced MRI of the breast. J Magn Reson Imaging 30, 999-1004, doi:10.1002/jmri.21947 (2009).

60 Escobar-Zepeda, A. et al. Analysis of sequencing strategies and tools for taxonomic annotation: Defining standards for progressive metagenomics. Sci Rep 8, 12034, doi:10.1038/s41598-018-30515-5 (2018).

61 Marotz, C. A. et al. Improving saliva shotgun metagenomics by chemical host DNA depletion. Microbiome 6, 42, doi:10.1186/s40168-018-0426-3 (2018).

62 Nelson, M. T. et al. Human and Extracellular DNA Depletion for Metagenomic Analysis of Complex Clinical Infection Samples Yields Optimized Viable Microbiome Profiles. Cell Rep 26, 2227-2240 e2225, doi:10.1016/j.celrep.2019.01.091 (2019).

63 Barlow, J. T., Bogatyrev, S. R. \& Ismagilov, R. F. A quantitative sequencing framework for absolute abundance measurements of mucosal and lumenal microbial communities. Nat Commun 11, 2590, doi:10.1038/s41467-020-16224-6 (2020).

64 Costea, P. I. et al. Towards standards for human fecal sample processing in metagenomic studies. Nat Biotechnol 35, 1069-1076, doi:10.1038/nbt.3960 (2017).

65 Pacholewicz, E. et al. Internal sample process control improves cultivation-independent quantification of thermotolerant Campylobacter. Food Microbiol 78, 53-61, doi:10.1016/j.fm.2018.09.017 (2019).

66 Hardwick, S. A. et al. Synthetic microbe communities provide internal reference standards for metagenome sequencing and analysis. Nat Commun 9, 3096, doi:10.1038/s41467-01805555-0 (2018).

67 Tourlousse, D. M. et al. Synthetic spike-in standards for high-throughput 16S rRNA gene amplicon sequencing. Nucleic Acids Res 45, e23, doi:10.1093/nar/gkw984 (2017).

68 Shen, J. \& Hartmann, E. M. Collect, extract, pool, and cultivate surface swab samples from built environments. Protocol Exchange, doi:10.21203/rs.3.pex-1656/v1 (2021).

69 Gomez-Silvan, C. et al. A comparison of methods used to unveil the genetic and metabolic pool in the built environment. Microbiome 6, 71, doi:10.1186/s40168-018-0453-0 (2018).

70 Shen, J. \& Hartmann, E. M. Snap freezing of environmental microbial samples in liquid. Protocol Exchange, doi:10.21203/rs.3.pex-1657/v1 (2021).

71 Wang, X., Howe, S., Deng, F. \& Zhao, J. Current Applications of Absolute Bacterial Quantification in Microbiome Studies and Decision-Making Regarding Different Biological Questions. Microorganisms 9, 1797 (2021). 
72 Fahimipour, A. K. et al. Daylight exposure modulates bacterial communities associated with household dust. Microbiome 6, 175, doi:10.1186/s40168-018-0559-4 (2018).

73 Shen, J., Rose, L. J., Perry-Dow, K. A. \& Hartmann, E. M. An optimized protocol for propidium monoazide treatment. Protocol Exchange, doi:10.21203/rs.3.pex-1659/v1 (2021).

74 Leung, M. H. Y. et al. Characterization of the public transit air microbiome and resistome reveals geographical specificity. Microbiome 9, 112, doi:10.1186/s40168-021-01044-7 (2021).

75 Shen, J. \& Hartmann, E. M. DNA extraction protocol for low-biomass environmental
samples: adapted from the Lucigen MasterPure Complete DNA and RNA Purification Kit manual. Protocol Exchange, doi:10.21203/rs.3.pex-1658/v1 (2021).

76 Singer, V. L., Jones, L. J., Yue, S. T. \& Haugland, R. P. Characterization of PicoGreen reagent and development of a fluorescence-based solution assay for double-stranded DNA quantitation. Analytical biochemistry 249, 228-238, doi:10.1006/abio.1997.2177 (1997). Klindworth, A. et al. Evaluation of general 16S ribosomal RNA gene PCR primers for classical and next-generation sequencing-based diversity studies. Nucleic Acids Res 41, e1, doi:10.1093/nar/gks808 (2013). Segata, N. et al. Metagenomic microbial community profiling using unique clade-specific marker genes. Nature Methods 9, 811-814, doi:10.1038/nmeth.2066 (2012). 
Fig. 1

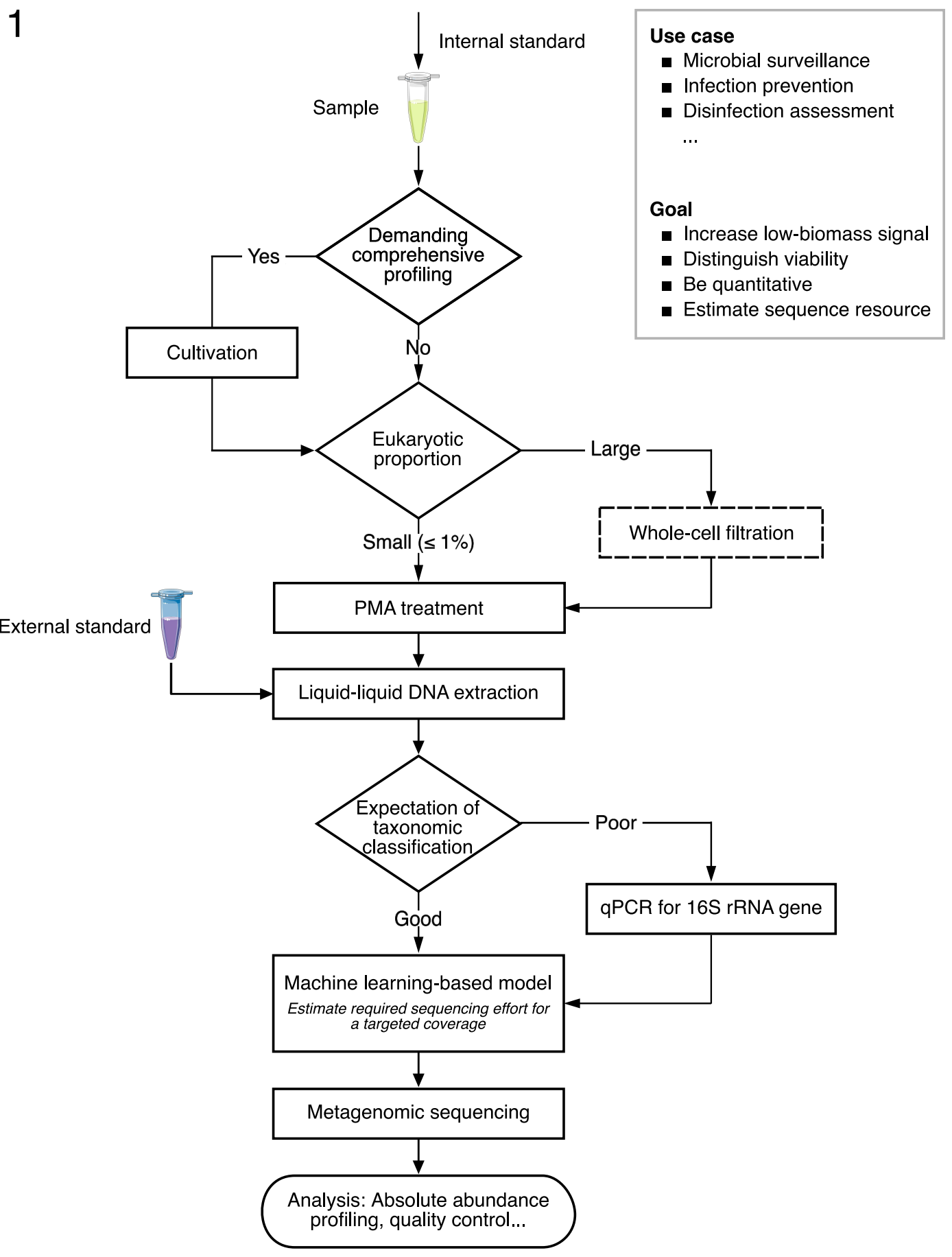

821 Figure 1: A workflow for metagenomics-based environmental surveillance that is appropriate for low-biomass samples, distinguishes viability, is quantitative, and estimates sequence resources. 
Fig. 2

a)

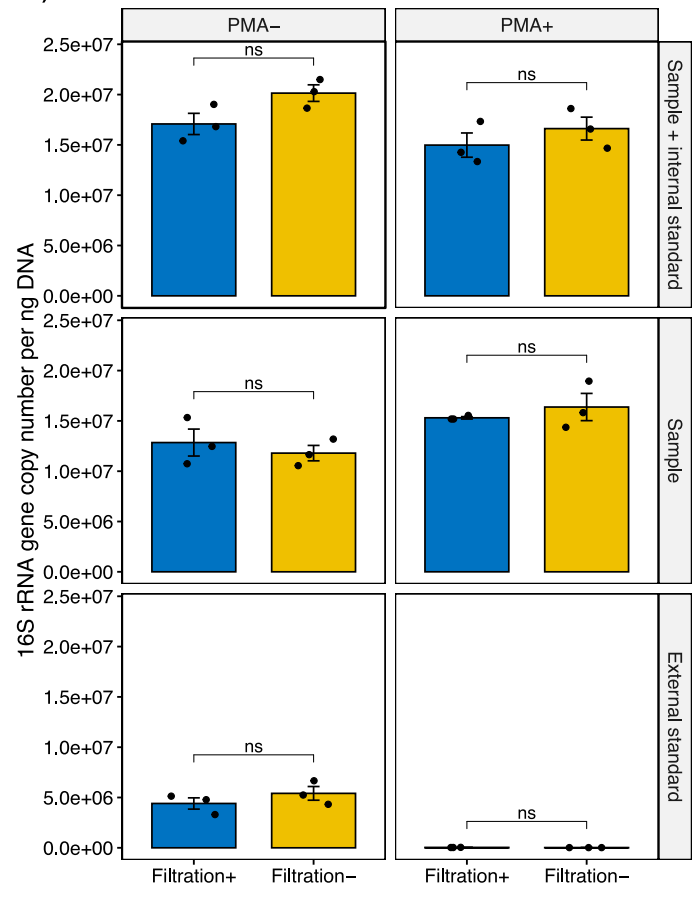

c)

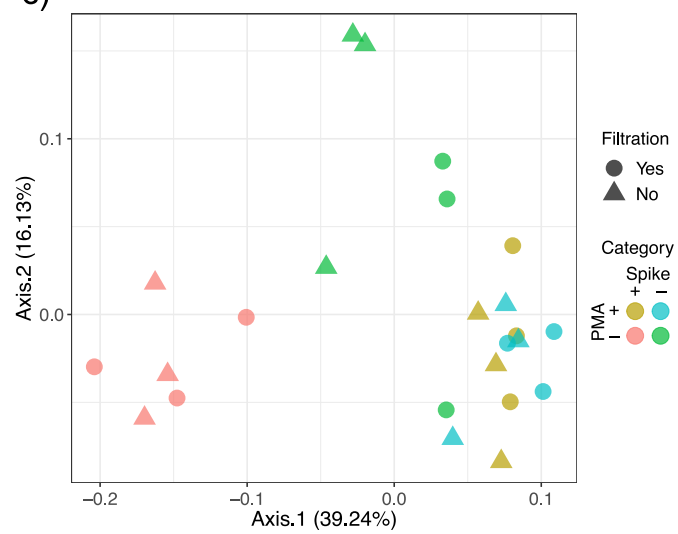

b)

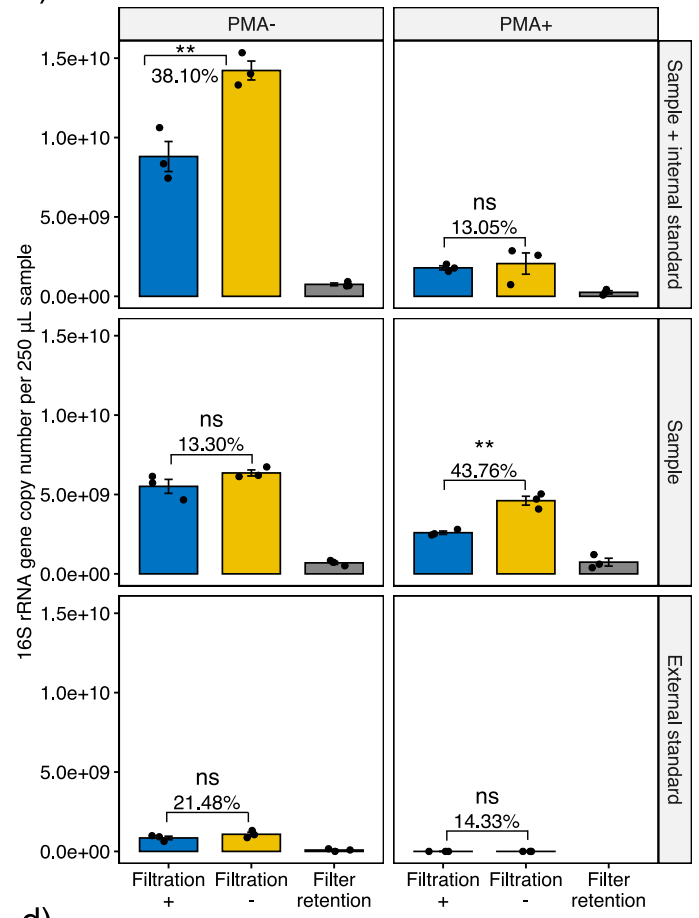

d)

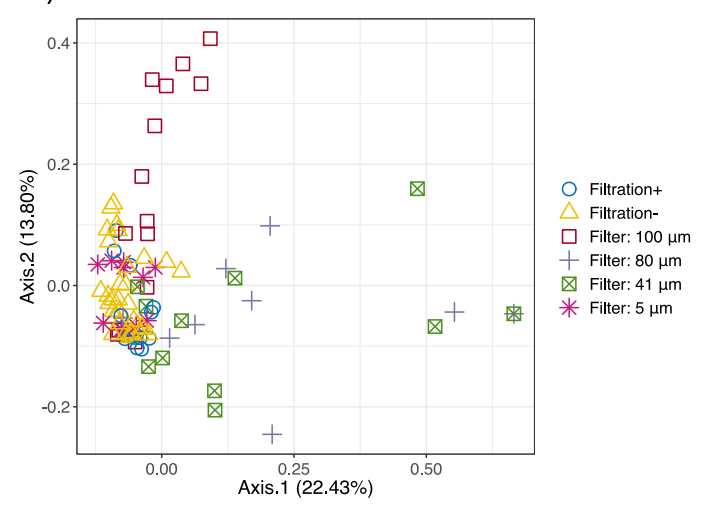

Figure 2: Effects of sequential filtration on hospital-associated surface samples. a) Bacteria proportion was not significantly increased after filtration according to paired $t$ tests. b) Biomass of samples with and without filtration as well as retained by filters according to $16 \mathrm{~S}$ rRNA gene copy number. In a) and b), error bars represent the mean standard error of triplicates. Filter retention includes all biomass captured by $100,80,41$, and $5 \mu \mathrm{m}$ filters. Ns and ** are significance codes, representing $\mathrm{p}>0.05$ and $0.001<\mathrm{p} \leq 0.01$, respectively. A linear scale was used for both a) and b) because for a), a linear scale is more conservative than a log scale when no significant difference was concluded; for b), linear-scale biomass loss is more informative for metagenomic sequencing. c) Principle coordinate analysis using Jaccard distance metric among samples with and without filtration. d) Principle coordinate analysis based on Jaccard distance metric revealed that bacterial profiles retained on $5 \mu \mathrm{m}$ filters clustered together with liquid samples, while those on 100, 80, and $41 \mu \mathrm{m}$ filters were away from the major group. 
Fig. 3

a)

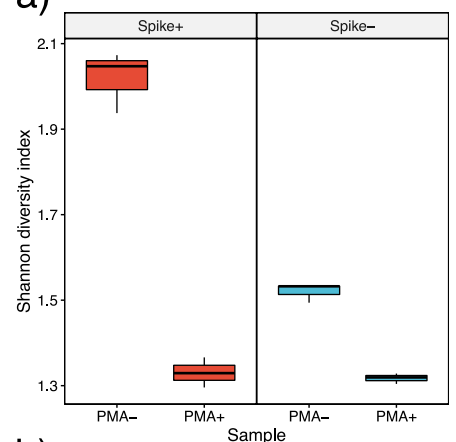

b)

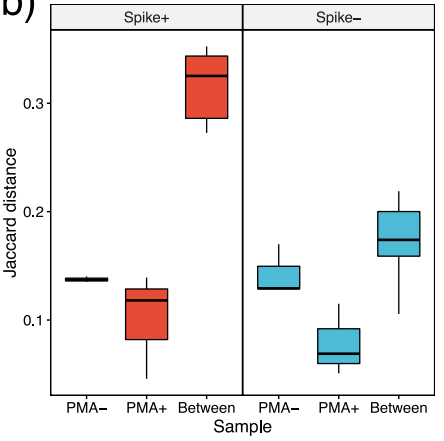

c)
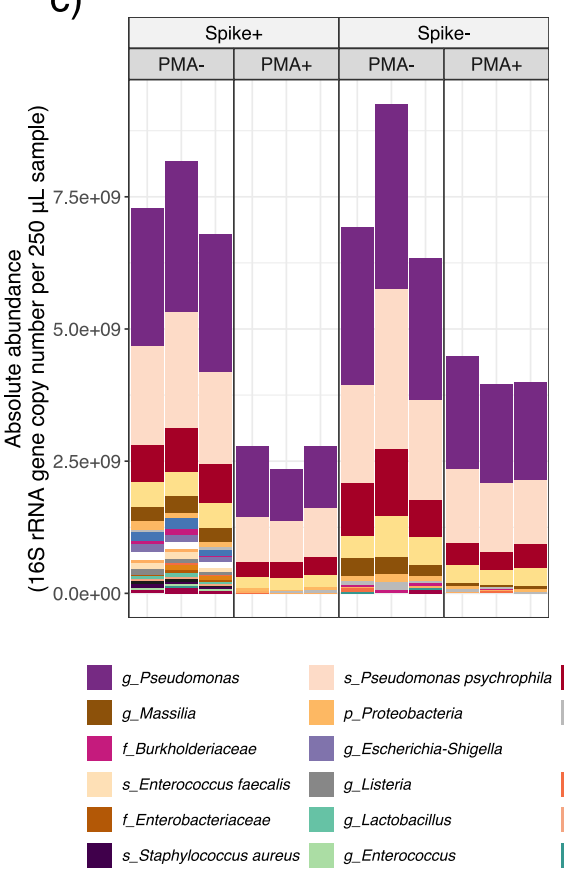

d)

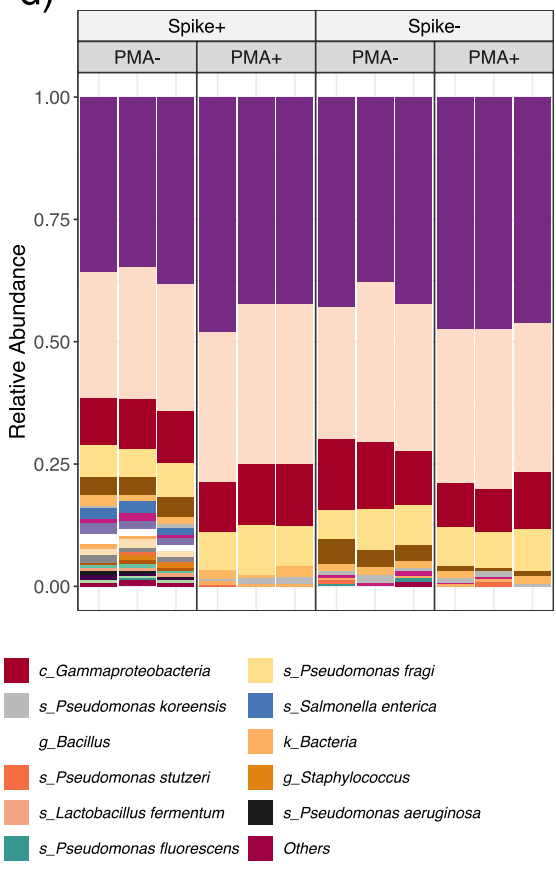

Figure 3: Effects of PMA treatment on hospital-associated surface samples. a) PMA treated samples had lower $\alpha$ composition by c) absolute abundance and c) relative abundance. 


\section{Fig. 4}

a)

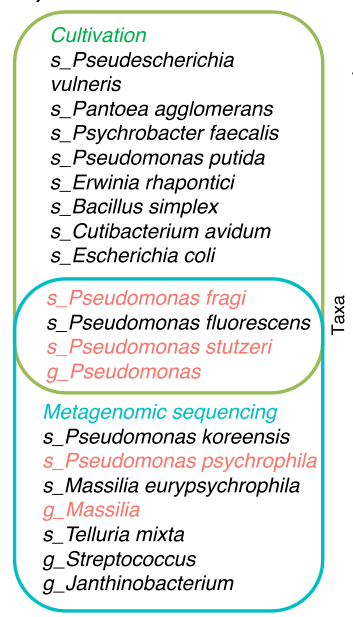

842

843

844

845

846

847

848 b)

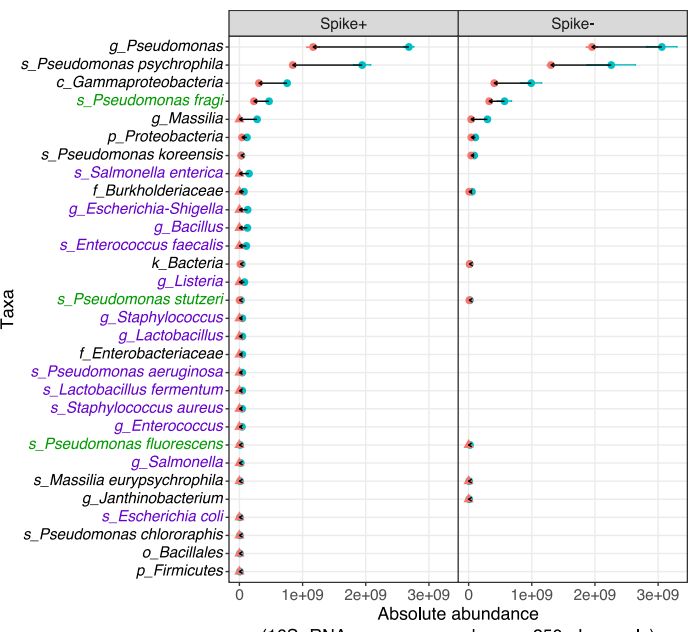

(16S rRNA gene copy number per $250 \mu \mathrm{L}$ sample)

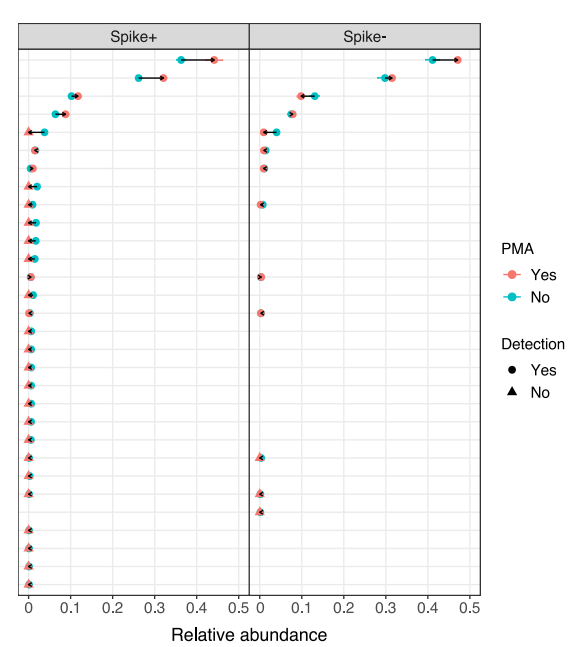

- Yes

- No

Detection

- Yes

Figure 4: Performance of cultivation and PMA-MetaSeq in viability distinction of hospital-associated surface samples. a) Venn diagram showing the detected taxa by cultivation and MetaSeq. Taxa detected by PMA-MetaSeq are color coded in red. b) The abundance change of all taxa detected under the framework of absolute abundance and relative abundance. Taxa in the theoretical composition of the internal standard and recovered in cultivation are color coded in purple and green, respectively. The $\mathrm{Y}$ axis follows a descending order of the average abundance across samples. Error bars represent the mean standard error of triplicates. 
Fig. 5

a)
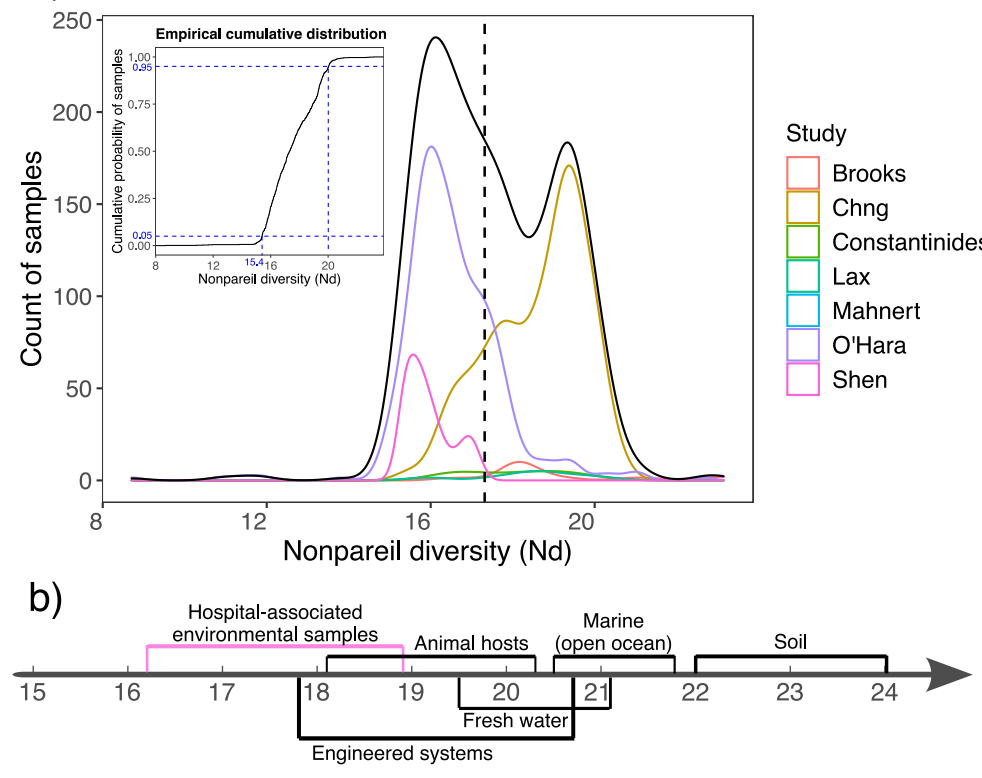

c)

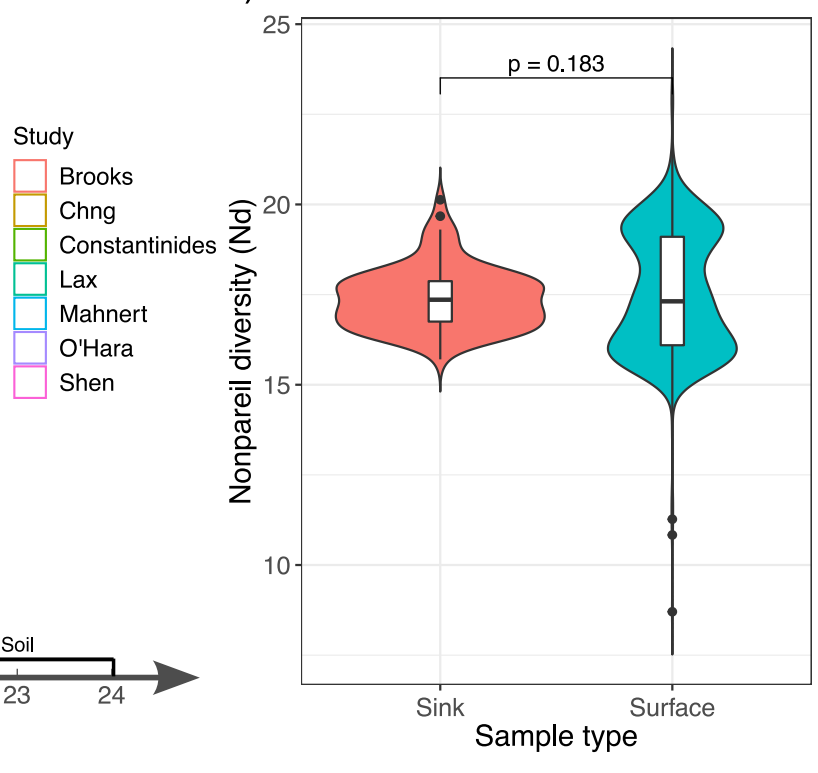

d)

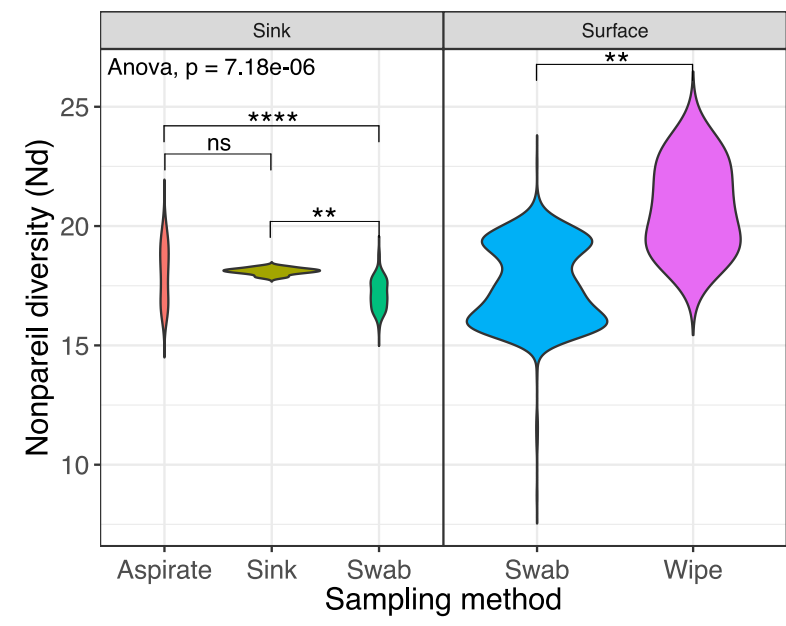

e)

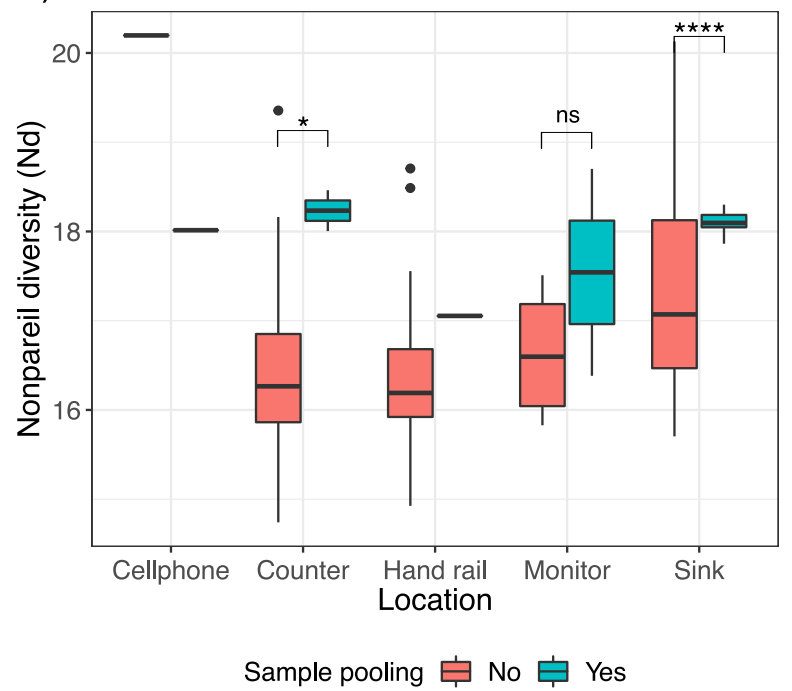

Figure 5: Relationships between Nonpareil diversity and metadata features for hospital-associated surface samples. a) Overall distribution of Nonpareil diversity (black) and distributions for individual studies. b) Interquartile range of Nonpareil diversity for microbiome samples from different environments. This study is color coded in orchid. Effects of c) sample type, d) sampling method, e) and sample pooling on Nonpareil diversity. Significance codes are: $\mathrm{p}>0.05(\mathrm{~ns}), 0.01<\mathrm{p} \leq 0.05(*), 0.001<\mathrm{p} \leq 0.01(* *), \mathrm{p} \leq 0.001(* * *)$. 


\section{Fig. 6}

a)
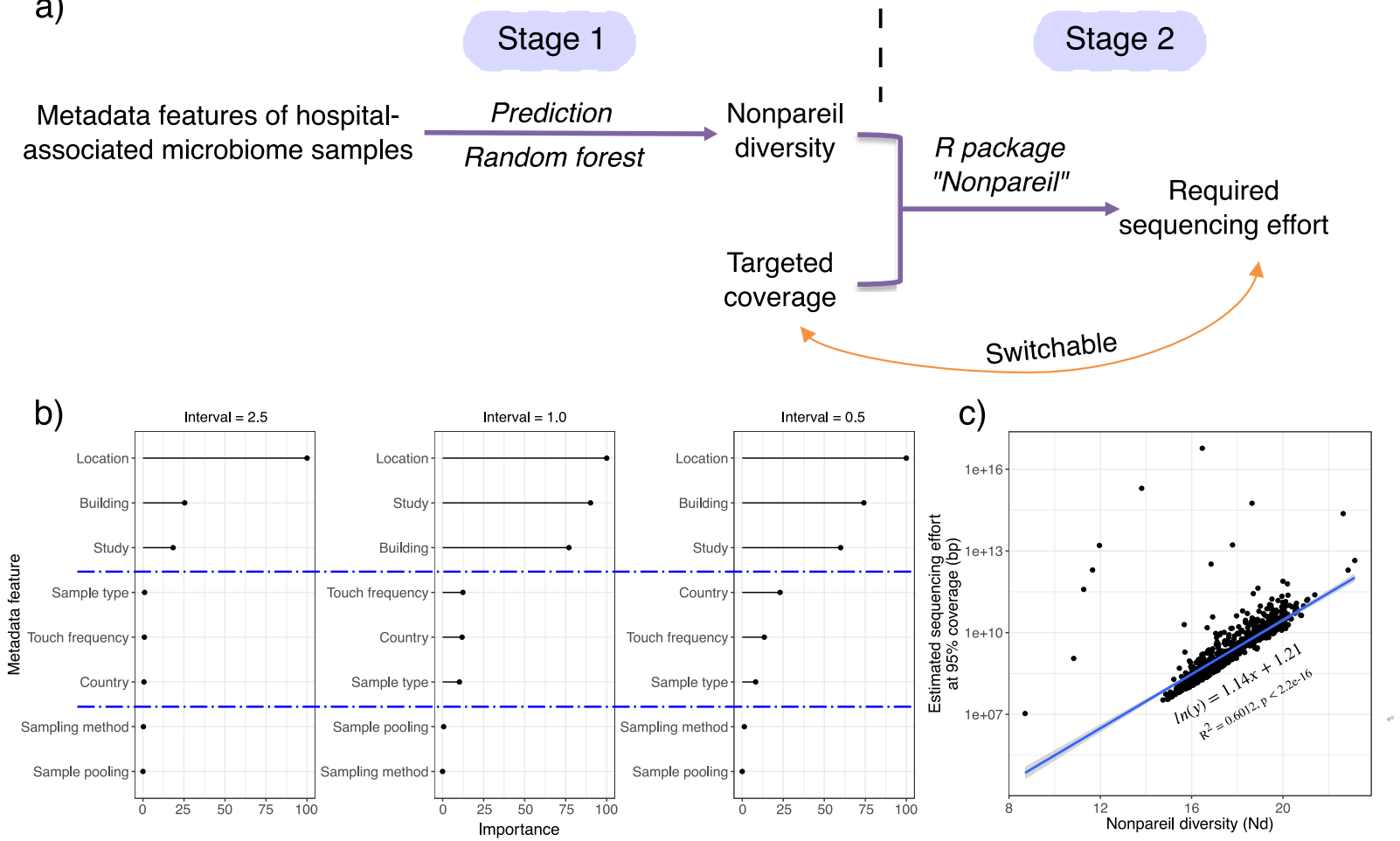

Figure 6: Required sequencing effort can be predicted by accessible sample features and targeted coverage. a) Workflow of making the prediction. b) Variable importance rankings by random forest. c) The natural log of estimated sequencing effort at $95 \%$ coverage is linearly correlated with Nonpareil diversity. 


\section{Supplementary Files}

This is a list of supplementary files associated with this preprint. Click to download.

- supt2samplingcondition.xIsx

- supt1predictiondataset.xlsx

- supplementaryfigurescombined.pdf 